\title{
ON THE EXISTENCE AND IRREDUCIBILITY OF CERTAIN SERIES OF REPRESENTATIONS ${ }^{1}$
}

\author{
BY BERTRAM KOSTANT
}

1. Introduction. 1. By the principal series one means here the unitary representations of a semisimple Lie group $G$ arising from induction to $G$ by characters on $M A N$ corresponding to characters on $A$. Although long conjectured to be irreducible, this family of representations has been shown to be irreducible only for special groups. For example see [9] for complex $G$ and see [3] for the group $\operatorname{Sl}(n, R)$. In the general case (all $G$ ) irreducibility has been proved by Bruhat [1] using analytic methods, only however, for the "regular" characters on $A$. A proof of the irreducibility of all the elements of the principal series is but one application of certain algebraic results, stated here, on modules of the universal enveloping algebra $U$ of the Lie algebra $g$ of $G$.

A second application is the proof of the existence and irreducibility of the complementary series for all semisimple Lie groups generalizing in a natural way the case of $\mathrm{Sl}(2, R)$. It is shown also that if $\operatorname{dim} A=1$ (split rank 1 case) then except for possibly the trivial (one dimensional) representation the most general irreducible unitary represensation of $G$ admitting a fixed vector for $K\left(\operatorname{Ad}_{\mathfrak{g}} K\right.$ is the maximal compact subgroup of $\operatorname{Ad}_{\mathfrak{g}} G$ ) belongs either to the principal or complementary series.

1.2. If $a_{\mathrm{C}}^{\prime}$ is the complex dual to the Lie algebra $a$ of $A$ then any $\lambda \in \mathfrak{a}_{\mathrm{C}}^{\prime}$ defines a one dimensional representation $b \rightarrow b^{\lambda}$ of $B=M A N$. If $X^{\lambda}$ is the space of all analytic $K$-finite functions $f$ on $G$ such that $f(a b)=b^{-\lambda} f(a)$ where $a \in G, b \in B$ then $X^{\lambda}$ is in a natural way a $U$ module. The results above are mainly applications of a theorem (Theorem 2) giving a necessary and sufficient condition on $\lambda$ for $X^{\lambda}$ to be an irreducible (in the usual algebraic sense) $U$-module. In particular there arises, in a natural way, a region in $a_{c}^{\prime}$ which we call the critical strip (CS) for which $X^{\lambda}$ is always $U$-irreducible. The critical strip contains all of the $\lambda$ corresponding to the principal series and its closure contains all the $\lambda$ corresponding to the complementary series.

${ }^{1}$ By invitation the author addressed the annual meeting of the American Mathematical Society in Cincinnati on January 22, 1962 on the topic A survey of Lie group representations. The present paper is partially an outgrowth of some of the ideas on multiplicities of representations stated during that talk; received by the editors March 3, 1969. 
1.3. Now $X^{\lambda}$ contains a unique function, $1_{\lambda}$, which is the constant 1 on $K$. In the general case $X^{\lambda}$ is not irreducible and in fact the cyclic $U$-submodule $X_{\theta}^{\lambda}=U \cdot 1_{\lambda}$ is not equal to $X^{\lambda}$. It is proved, however, that if $C$ is the complex Weyl chamber, suitably translated, then $X_{\theta}^{\lambda}$ is $U$-irreducible for all $\lambda \in-C$ and that every irreducible $U$-module $Z$ such that $Z^{\mathfrak{t}} \neq 0$ ( $\mathfrak{f}$ is the Lie algebra of $K$ ) is equivalent to $X_{e}^{\lambda}$ for some $\lambda \in-C$. On the other hand, if $\lambda \in C$ one always has $X_{e}^{\lambda}=X^{\lambda}$ and that every $U$-module $Y$ cyclically generated by an element in $Y^{\mathfrak{t}}$, where $\operatorname{dim} Y^{\mathfrak{l}}=1$, is equivalent to a quotient of $X^{\lambda}$ for some $\lambda \in C$.

The completeness result on the unitary representations of $G$ in the split rank 1 case is a consequence of another theorem which asserts that in the split rank 1 case if $G$ is chosen as defined in $\$ 4.1$ the multiplicity of any irreducible representation of $K$ in $X^{\lambda}$, for any $\lambda \in \mathfrak{a}_{\mathrm{C}}^{\prime}$, is at most one.

2. The principal series. 1 . Let $G$ be a Lie group, not necessarily connected. Let $\mathfrak{g}$ be its Lie algebra, so that $\mathfrak{g}$ is a Lie algebra over $\boldsymbol{R}$. Assume that $\mathfrak{g}$ is semisimple and assume that for any $a \in G$ the operator Ad $a$ on the complexification of $g_{\mathrm{c}}$ of $\mathfrak{g}$, defined by conjugation of $G$ by $a$, lies in the adjoint group of gc.

REMARK 1. The second assumption is of course satisfied if $G$ is connected. However it is also satisfied if, for example, $G$ is the set of rational points of a complex connected algebraic group defined over $R$.

We recall that a subalgebra $\mathfrak{b} \subseteq \mathfrak{g}$ is called parabolic in case the complexification of $\mathfrak{b}$ contains a maximal solvable subalgebra (a Borel subalgebra) of $\mathfrak{g}_{\mathrm{C}}$.

Let $\mathfrak{b} \subseteq \mathfrak{g}$ be a minimal parabolic subalgebra of $\mathfrak{g}$ (all such are conjugate) and let $\mathfrak{n}$ be the nil-radical of $\mathfrak{b}$. Then $\mathfrak{b}$ can be written as a semidirect sum $\mathfrak{b}=\mathfrak{h}+\mathfrak{n}$ where the subalgebra $\mathfrak{h} \subseteq \mathfrak{b}$ is unique up to conjugacy.

An element $x \in \mathfrak{g}$ is called real semisimple in case ad $x$ is diagonizable on $\mathfrak{g}$ (and hence real eigenvalues).

Now let $\mathfrak{a}$ be the set of all elements in the center of $\mathfrak{h}$ which are real semisimple. Then one knows that $\mathfrak{a}$ is an abelian subalgebra of $\mathfrak{g}$ maximal with the property that all of its elements are real semisimple. Furthermore if $A \subseteq G$ is the corresponding subgroup then $A$ is simply connected and hence $A \cong R^{r}$ where $r=\operatorname{dim} a$.

Now let $G^{A}$ be the centralizer of $A$ in $G$ and let $N \subseteq G$ be the subgroup corresponding to $\mathfrak{n}$. Then one knows that $G^{A}$ normalizes $N$ and $G^{A} \cap N=(e)$. Let $B=G^{A} N$. Then the Lie algebra of $B$ is $\mathfrak{b}$. Moreover there exists a closed subgroup $M \subseteq G^{A}$ such that $G^{A}$ is a direct product 
$G^{A}=M A$. Moreover if $D$ (= $\left.\operatorname{Ker} A d\right)$ is the centralizer of $G_{0}$ in $G$ ( $G_{e}$ is the identity component of $G$ ) then $D$ is discrete, normal and $\mathfrak{a}$ can be chosen so that $D \subseteq M$. With this additional property $M$ is unique and we shall assume that $M$ is so chosen. One thus has $B=M A N$.

Now let $\mathfrak{a}_{\mathbf{C}}^{\prime}=\operatorname{Hom}_{\mathbb{R}}(\mathfrak{a}, \mathbf{C})$ be the complex dual to $\mathfrak{a}$. Any $\lambda \in \mathfrak{a}_{\mathbf{C}}^{\prime}$ defines a character $b \rightarrow b^{\lambda}$ on $B$ as follows: For $b \in M N$ put $b^{\lambda}=1$. If $b \in A$ then one may uniquely write $b=\exp x$, for $x \in \mathfrak{a}$. Put $b^{\lambda}=\exp \langle\lambda, x\rangle$. One obtains in this way all complex characters on $B$ which are trivial on $M$.

2.2. We will be concerned with the representations of $G$ induced by the one dimensional representations of $B$ given by $b \rightarrow b^{\lambda}, \lambda \in \mathfrak{a}_{\mathbf{c}}^{\prime}$. Let $\lambda \in \mathfrak{a}_{\mathrm{c}}^{\prime}$ and let $X^{(\lambda)}$ be the space of all measurable functions $f$ on $G$ such that $f(a b)=b^{-\lambda} f(a)$ for all $a \in G, b \in B$. Then $X^{(\lambda)}$ is a $G$ module where if $a \in G$ and $f \in X^{(\lambda)}$ then $a \cdot f \in X^{(\lambda)}$ is given by $(a \cdot f)(g)$ $=f\left(a^{-1} g\right)$ for all $g \in G$.

Now for certain $\lambda$ one obtains a unitary representation: Indeed we first observe that $G / D$ is a centerless Lie group with at most a finite number of connected components. As such it contains a maximal compact subgroup $K_{0}$, unique up to conjugacy. Let $K \subseteq G$ be the unique subgroup of $G$ containing $D$ and such that $K / D=K_{0}$. Let $\mathfrak{l} \subseteq \mathfrak{g}$ be the Lie algebra of $K$ so that $\mathfrak{l}$ corresponds to a maximal compact subgroup of the adjoint group of $g$. We can choose $K_{0}$, and do so, so that the Cartan involution of $\mathfrak{g}$ defined by $\mathfrak{l}$ leaves $\mathfrak{h}$, the fixed reductive part of $\mathfrak{b}$, stable.

Now let $L=K \cap M$. Then the $K$-homogeneous space $K / L$ is connected compact and has a unique $K$-invariant measure $\omega$ of total volume 1 . Now let $\mathfrak{a}^{\prime} \subseteq \mathfrak{a}_{\mathrm{c}}^{\prime}$ be the real dual to $\mathfrak{a}$ and let $\rho \in \mathfrak{a}^{\prime}$ be defined by $\langle\rho, x\rangle=\frac{1}{2} \operatorname{tr}$ ad $x \mid \mathfrak{n}$ for all $x \in \mathfrak{a}$.

Now if $\lambda \in \mathfrak{a}_{\mathrm{c}}^{\prime}$ is of the form $\lambda=\rho+i \nu$ where $\nu \in \mathfrak{a}^{\prime}$, let $\mathfrak{F C}_{\nu}$ be the Hilbert space of all $f \in X^{(\lambda)}$ such that

$$
\int_{K / L}|f|^{2} \omega<\infty .
$$

The integral is well defined since $f \mid K$ is constant on the left cosets of $L$ in $M$. Furthermore $\mathcal{F C}_{\nu}$ is stable under the action of $G$ defining a representation $s_{\nu}$ of $G$ on $\mathfrak{F C}_{\nu}$ which is in fact unitary.

The family or series of unitary representations $s_{\nu}$ obtained as $\nu$ runs through $\mathfrak{a}^{\prime}$ is called the principal series of class zero or simply, in our terminology, principal series.

It has been long conjectured that all members of the principal 
series are irreducible. For certain special groups (e.g. some classical groups) this has been proved. Using analytic methods (e.g. the Schwartz-Kernel theorem) Bruhat has proved irreducibility for the case where $\nu$ is a regular element of $\mathfrak{a}^{\prime}$. If $G$ is complex, Parthasarathy, Rao and Varadarajan [9] have proved irreducibility for all $\nu$. Based on a similar approach we can prove

THEOREM 1. For all $G$ every member of the principal series $s_{\nu}$ is an irreducible unitary representation. Moreover $s_{\nu}$ is equivalent to $s_{\nu_{1}}$ if and only if $\nu$ and $\nu_{1}$ are conjugate under the Weyl group.

3. The $U$-module and the critical strip. 1 . Theorem 1 is an application of the much more complete result which follows: The method used in the proof is purely algebraic and is modeled after [9] in the sense that it involves a detailed examination of a family of polynomial valued matrices. The result rests upon [6], [7] in the same sense that [9] rests upon [5].

Now for any $\lambda \in \mathfrak{a}_{\mathrm{c}}^{\prime}$ let $X^{\lambda}$ be the subspace of all $f \in X^{(\lambda)}$ which are $K$-finite, i.e., $K \cdot f$ spans a finite dimensional space. The elements of $X^{\lambda}$ are analytic functions (real sense) in $G$ and although $X^{\lambda}$ is no longer in general a $G$-module it is, however, a $U$-module where $U$ is the universal enveloping algebra of $\mathfrak{g}$ over C. Indeed if $f \in X^{\lambda}$ then $x \cdot f \in X^{\lambda}$ where $x \in \mathfrak{g}$ and $x \cdot f$ is the function on $G$ given by

$$
x \cdot f(g)=\left.\frac{d}{d t} f(\exp -t x \cdot g)\right|_{t=0} .
$$

The next result gives the set of all $\lambda \in \mathfrak{a}_{\mathrm{c}}^{\prime}$ such that $X^{\lambda}$ is an irreducible (in the usual algebraic sense) $U$-module.

Let $\Lambda \subseteq \mathfrak{a}^{\prime}$ be the set of (sometimes referred to as restricted) roots for the action of $a$ on $\mathfrak{g}$ and for any $\phi \in \Lambda$ let $\mathfrak{g}^{\phi} \subseteq \mathfrak{g}$ be the corresponding root space. A lexicographical ordering may be introduced into $\mathfrak{a}^{\prime}$ so that if $\Lambda_{+}$is the set of positive roots then

$$
\mathfrak{n}=\sum_{\phi \in \Lambda_{+}} \mathfrak{g}^{\phi}
$$

Now for any $\phi \in \Lambda$ the space $\left[\mathfrak{g}^{\phi}, \mathfrak{g}^{-\phi}\right] \cap \mathfrak{a}$ is one dimensional and is spanned by a unique element $w_{\phi}$ such that $\left\langle\phi, w_{\phi}\right\rangle=1$. If $\phi \in \Lambda$ then $\phi / 2$ or $2 \phi$ may be a root. Let $\Lambda^{1}$ be the set of all $\phi \in \Lambda$ such that $\phi / 2$ is not a root (the set $\Lambda^{1}$ may be characterized as the set of roots which may be embedded in some simple system) and let $\Lambda_{+}^{1}$ be the positive elements in $\Lambda^{1}$. Of course if $\phi \in \Lambda_{+}^{1}$ then $2 \phi$ may be a root.

For any $\phi \in \Lambda_{+}^{1}$ let $T_{\phi} \subset R$ be the open interval, symmetric about 
the origin, given by $T_{\phi}=\left\{t \in R|| t \mid<\operatorname{dim} g^{\phi} / 2\right.$ if $2 \phi$ is not a root and $|t|<\operatorname{dim} g^{\phi} / 2+1$ if $2 \phi$ is a root $\}$.

We now define a region in $\mathfrak{a}_{\mathbf{C}}^{\prime}$, symmetric around the point $\rho$, which arises in a natural way from our theory and which plays a special role in the representation theory. We will call the region the critical strip, CS, and it is defined by

$$
\mathrm{CS}=\left\{\lambda \in a_{\mathbf{C}}^{\prime} \mid \operatorname{Re}\left\langle\lambda-\rho, w_{\phi}\right\rangle \in T_{\phi} \text { for all } \phi \in \Lambda_{+}^{1}\right\} .
$$

For $\operatorname{Sl}(2, R)$ it reduces to the strip of all $z \phi, z \in \mathbf{C},(\phi)=\Lambda_{+}^{1}$, such that $0<\operatorname{Re} z<1$.

Now for any root $\phi \in \Lambda_{+}^{1}$ let $m_{\phi}$ be the integer or half-integer given by $m_{\phi}=\left(\operatorname{dim} \mathfrak{g}^{\phi} / 2\right)+\operatorname{dim} \mathfrak{g}^{2 \phi}$ and let $n_{\phi}=1$ if $2 \phi$ is not a root and $n_{\phi}=2$ if $2 \phi$ is a root.

It is somewhat easier to describe the set of all $\lambda \in a_{c}^{\prime}$ such that $X^{\lambda}$ is not $U$-irreducible.

THEOREM 2. Let $\lambda \in a_{\mathrm{C}}^{\prime}$ be arbitrary then the $U$-module $X^{\lambda}$ is not irreducible if and only if there exists a root $\phi \in \Lambda_{+}^{1}$ such that

(1) $\left\langle\lambda-\rho, w_{\phi}\right\rangle \notin T_{\phi}$ and

(2) $\left\langle\lambda-\rho, w_{\phi}\right\rangle+m_{\phi}=0 \bmod n_{\phi} Z$.

In particular $X^{\lambda}$ is $U$-irreducible whenever $\lambda$ lies in the critical strip CS.

3.2. A $U$-module $X$ will be said to be admissible in case $X^{\mathfrak{l}}$, the annihilator of $\mathfrak{t}$ in $X$, is one dimensional. Let $\mathfrak{e}$ be the set of $U$ equivalence classes $\{X\}$ of all admissible $U$-modules $X$.

Now for any $u \in U$ one knows there exists a unique element $p^{u} \in U(\mathfrak{a})$, (the enveloping algebra of $\mathfrak{a}$ ) such that $u-p^{u} \in \mathfrak{n} U+U \mathfrak{l}$. Also if $U^{\mathfrak{t}}$ is the centralizer of $\mathfrak{l}$ in $U$ then the map $u \rightarrow p^{u}$ is a homomorphism on $U^{t}$. Now regarding $U(\mathfrak{a})$ as the polynomial ring on $\mathfrak{a}_{\mathbf{c}}^{\prime}$ let $\mathfrak{e}_{\lambda} \subseteq \mathfrak{e}$, for any $\lambda \in \mathfrak{a}_{\mathbf{C}}^{\prime}$, be the set of all $\{X\} \in \mathcal{e}$ such that $u \cdot \xi$ $=p^{u}(\lambda) \xi$ for all $u \in U^{\mathfrak{t}}$ where $X \mathfrak{t}=(\xi)$.

Now if $W$ is the Weyl group operating in $a_{C}^{\prime}$ let $\tilde{W}$ be the group of affine transformations in $a_{c}^{\prime}$ of the form $\lambda \rightarrow \tilde{\sigma} \lambda=\sigma(\lambda-\rho)+\rho$ where $\sigma \in W$. One has

Proposition 1. $\mathfrak{e}$ is a union of $\mathfrak{C}_{\lambda}$ over all $\lambda \in a_{\mathbf{C}}^{\prime}$. Moreover if $\lambda$, $\nu \in \mathfrak{a}_{\mathrm{C}}^{\prime}$ then $\mathcal{C}_{\lambda}$ and $\mathfrak{C}_{\nu}$ are either disjoint or equal and they are equal if and only if $\lambda$ and $\nu$ are $\tilde{W}$ conjugate.

REMARK 2. One readily shows that for all $\lambda \in a_{\mathbf{c}}^{\prime}$ one has $\left\{X^{\lambda}\right\} \in \mathfrak{C}_{\lambda}$. 3.3. With regard to irreducibility one has

Proposition 2. For any $\lambda \in \mathfrak{a}_{\mathrm{C}}^{\prime}$ there exist a unique element $\left\{Z^{\lambda}\right\} \in \mathcal{C}_{\lambda}$ 
such that $Z^{\lambda}$ is $U$-irreducible. Moreover any irreducible $U$-module $Z$ such that $Z^{\mathfrak{t}} \neq 0$ is admissible and hence is equivalent to some $Z^{\lambda}$ where $\lambda$ is unique up to $\tilde{W}$ conjugacy in $\mathfrak{a}_{\mathrm{c}}^{\prime}$.

For any $\lambda \in \mathfrak{a}_{\mathbf{C}}^{\prime} Z^{\lambda}$ may be explicitly given by putting $Z^{\lambda}=U / L_{\lambda}$ where $L_{\lambda}$ is the left ideal in $U$ defined by

$$
L_{\lambda}=\left\{u \in U \mid p^{(v u)_{0}}(\lambda)=0 \text { for all } v \in U\right\} .
$$

Here $w_{0} \in U^{k}$, for any $w \in U$, is the component of $w$ in $U^{t}$ corresponding to the direct sum $U=[k, U]+U^{k}$.

REMARK 3. Note that by Theorem 2 one has that $\left\{X^{\lambda}\right\}=\left\{Z^{\lambda}\right\}$ if and only if $\lambda$ does not satisfy the condition of Theorem 2 . In particular the equality holds if $\lambda \in C S$.

3.4. Now let $u \rightarrow u^{s}$ be the conjugate-linear, antilinear $\left((u v)^{s}=v^{s} u^{s}\right)$ involution of $U$ satisfying $x^{s}=-x$ for $x \in g$. A Hermitian bilinear form $((\xi, \eta)=(\overline{\eta, \xi}), c(\xi, \eta)=(c \xi, \eta)=(\xi, \bar{c} \eta))$ on a $U$-module $X$ will be said to be $U$-invariant in case $(u \xi, \eta)=\left(\xi, u^{s} \eta\right)$ for all $u \in U, \xi, \eta \in X$. If $\lambda \in \mathfrak{a}_{\mathrm{c}}^{\prime}$ its conjugate $\bar{\lambda} \in \mathfrak{a}_{\mathbf{c}}^{\prime}$ is defined relative to the real form $\mathfrak{a}^{\prime}$ of $\mathfrak{a}_{\mathbf{c}}^{\prime}$.

Proposition 3. Let $\lambda \in \mathfrak{a}_{\mathrm{C}}^{\prime}$. Then there exists a nontrivial $U$-invariant Hermitian bilinear form on $Z^{\lambda}$ if and only if $\lambda$ and $-\bar{\lambda}+2 \rho$ are $\tilde{W}$ conjugate. Moreover in this case the form is unique up to a real scalar.

If $\lambda$ and $-\bar{\lambda}+2 \rho$ are $\tilde{W}$ conjugate then the $U$-invariant Hermitian form may be given explicitly by

$$
\left(u \cdot \xi_{\lambda}, v \cdot \xi_{\lambda}\right)=p^{\left(v s_{u}\right)_{0}}(\lambda)
$$

where $u, v \in U$ and $\xi_{\lambda} \in\left(Z^{\lambda}\right)^{k}$ is the image of 1 in $Z^{\lambda}=U / L_{\lambda}$. We denote the form by $B_{\lambda}$ and we will be concerned as to when it is positive definite so that Nelson's theorem, [8], can be applied.

4. The polynomial valued matrices $P^{r}, Q^{r}$ and $R^{r}$. 1. Now let $G_{\theta}$ be the set of all elements of the adjoint group of $g_{c}$ leaving $g$ stable. Note that the considerations above may be applied to $G_{\theta}$ instead of $G$. We let $K_{\theta}$ and $M_{\theta}$ be the subgroups of $G_{\theta}$ corresponding to the subgroups $K$ and $M$ of $G$. Also let $\Gamma$ be the set of all equivalence classes $\{V\}$ of all irreducible (finite dimensional, continuous) $K_{\theta^{-}}$ modules $V$ such that $V^{M_{\theta}} \neq 0$.

For any $\gamma \in \Gamma$ fix a $K_{\theta}$-module $V_{\gamma}$ in the class $\gamma$ and let $l(\gamma)=\operatorname{dim} V_{\gamma}^{M_{\theta}}$.

Also let $H \subseteq U$ be the subspace spanned by all powers $x^{j}, j=0$, $1, \cdots$, for all nilpotent elements $x$ in the complexification $p_{c}$ of $\mathfrak{p}$. Then if $\gamma \in \Gamma$ is arbitrary one knows from [6] that $\operatorname{dim} E_{\gamma}=l(\gamma)$ where $E_{\gamma}=\operatorname{Hom}_{K_{\theta}}\left(V_{\gamma}, H\right)$. 
Now if $v_{i}, i=1,2, \cdots, l(\gamma)$, is a basis of $V_{\gamma}^{M_{\theta}}$ and $\epsilon_{j}, j=1,2$, $\cdots, l(\gamma)$, is a basis of $E_{\gamma}$ we define an $l(\gamma) \times l(\gamma)$ matrix $P^{\gamma}$ with coefficients in $U(a)$ by defining $P_{i j}^{\gamma}=p^{\epsilon_{j}\left(v_{i}\right)}$. It is clear that a change of basis of $E_{\gamma}$ and $V_{\gamma}^{M_{\theta}}$ only changes $P^{\gamma}$ into a matrix of the form $A P^{r} B$ where $A$ and $B$ are nonsingular matrices with coefficients in C. In particular $p^{\gamma}=\operatorname{det} P^{\gamma}$ is an element of $U(\mathfrak{a})$ which, up to a scalar multiple, is independent of the choice of the bases. Also for any $\lambda \in \mathfrak{a}_{\mathbf{c}}$ the rank of the scalar matrix $\operatorname{Pr}(\lambda)$ is also independent of the basis.

Now if $\lambda \in \mathfrak{a}_{c}^{\prime}$ then the induced module $X^{\lambda}$ is not always $U$-irreducible (see Theorem 2). In fact if $1_{\lambda}$ is the unique element of $X^{\lambda}$ which as a function on $G$ equals the constant 1 on $K$ (and hence is a generator of $\left.\left(X^{\lambda}\right)^{t}\right)$ then in general the cyclic submodule $U \cdot 1_{\lambda}$ $=X_{e}^{\lambda} \subseteq X^{\lambda}$ is not equal to $X^{\lambda}$. A measure of its failure to equal $X^{\lambda}$ is given in the next proposition and is expressed in terms of the matrices $P^{r}$.

If $\gamma \in \Gamma$ and $X_{\gamma}^{\lambda}$ is the finite dimensional space of all $f \in X^{\lambda}$ which transforms under $K_{\theta}$ according to $\gamma$ then it is obvious that $X^{\lambda}$ is a direct sum of $X_{\gamma}^{\lambda}$ over all $\gamma$. The same is true of $X_{\theta}^{\lambda}$. In $X^{\lambda}$ the multiplicity of $\gamma$ is obviously $l(\gamma)$. In $X_{e}^{\lambda}$, however, the question of multiplicity is less trivial.

Proposition 4. For any $\lambda \in \mathfrak{a}_{\mathrm{c}}^{\prime}$ and $\gamma \in \Gamma$ the multiplicity of $\gamma$ in the $U$-cyclic submodule $U \cdot 1_{\lambda}=X_{e}^{\lambda}$ of the induced module $X^{\lambda}$ is exactly the rank of the matrix $P^{r}(\lambda)$.

In particular one has

Corollary to Proposition 4. For any $\lambda \in \mathfrak{a}_{\mathrm{C}}^{\prime}$ one has $X^{\lambda}=U \cdot 1_{\lambda}$ if and only if $p^{r}(\lambda) \neq 0$ for all $\gamma \in \Gamma$.

4.2. Now for any $\gamma \in \Gamma$ one defines another $l(\gamma) \times l(\gamma)$ matrix $Q^{\gamma}$ with coefficients in $U(\mathfrak{a})$ by the relation $Q^{\gamma}(\lambda)=P^{\gamma}(-\bar{\lambda}+2 \rho)^{*}$ for all $\lambda \in a_{\mathrm{C}}^{\prime}$. Here ${ }^{*}$ denotes the Hermitian adjoint of the matrix.

Now finally let $R^{r}$ be the $l(\gamma) \times l(\gamma)$ matrix with coefficients in $U(\mathfrak{a})$ defined by $R^{r}=Q^{r} P^{\gamma}$. Now whereas the $P^{\gamma}$ describe the structure of $U$-module $X_{e}^{\lambda}$ the $R^{r}$ describe the structure of the irreducible $U$ modules $Z^{\lambda}$. Moreover it determines the signature of the $U$-invariant Hermitian structure on $Z^{\lambda}$ when such a structure exists (that is when $\lambda$ and $-\bar{\lambda}+2 \rho$ are $\tilde{W}$ conjugate). Also $R^{\gamma}$ is invariant under the action of $\tilde{W}$.

The $U$-modules $Z^{\lambda}$ are in a natural way $K_{\theta}$-modules since the ideals $L_{\lambda} \subseteq U$ are stable under the adjoint action of $K_{\theta}$ on $U$. It follows that $Z^{\lambda}$ is a direct sum of the finite dimensional subspaces $Z_{\gamma}^{\lambda}, \gamma \in \Gamma$, of 
all elements in $Z^{\lambda}$ which transform under $K_{\theta}$ according to the representation class $\gamma$.

Proposition 5. For any $\lambda \in a_{\mathrm{C}}^{\prime}, \gamma \in \Gamma$, the multiplicity of $\gamma$ in the irreducible $U$-module $Z^{\lambda}$ is exactly the rank of the matrix $R^{r}(\lambda)$. Moreover for any $\sigma \in W$ one has $R^{\gamma}(\lambda)=R^{\gamma}(\tilde{\sigma} \lambda)$.

Finally if $\lambda$ and $-\bar{\lambda}+2 \rho$ are $\tilde{W}$ conjugate (so that the Hermitian bilinear form $B_{\lambda}$ on $Z^{\lambda}$ (see \$3.4) is defined) then $R^{\gamma}(\lambda)$ is a Hermitian matrix. Furthermore $R^{\gamma}(\lambda)$ is positive semidefinite if and only if $B_{\lambda} \mid Z_{\gamma}^{\lambda}$ is positive definite. In particular then (since the $Z_{\gamma}^{\lambda}$, over all $\gamma \in \Gamma$, are in any case $B_{\lambda}$ orthogonal) $B_{\lambda}$ defines a pre-Hilbert space structure on $Z^{\lambda}$ if and only if $R^{\gamma}(\lambda)$ is positive semidefinite for all $\gamma \in \Gamma$.

Now for any $\gamma \in \Gamma$ let $r^{\gamma}=\operatorname{det} R^{\gamma}$. One thus has that $r^{\gamma}(\lambda) \neq 0$ for all $\gamma$ if and only if $\left(Z^{\lambda}\right)=\left(X^{\lambda}\right)$. That is, one has

Corollary to Proposition 5. For any $\lambda \in \mathfrak{a}_{\mathrm{C}}^{\prime}$ the induced $U$-module $X^{\lambda}$ is $U$-irreducible if and only if $r^{\gamma}(\lambda) \neq 0$ for all $\gamma \in \Gamma$.

Let $\gamma \in \Gamma$ be arbitrary and put $q^{\gamma}=\operatorname{det} Q^{\gamma}$ so that $r^{\gamma}=q^{\gamma} p^{\gamma}$. But since $Q^{r}(\lambda)=P^{r}(-\bar{\lambda}+2 \rho)^{*}$ one has that

$$
r^{\gamma}(\lambda)=\overline{p^{\gamma}}(-\lambda+2 \rho) p^{\gamma}(\lambda)
$$

where conjugation in $U(\mathfrak{a})$ is the conjugate linear automorphism of $U(\mathfrak{a})$ which is the identity on $\mathfrak{a}$. The determination of the $r^{\gamma}$ thus depends upon the knowledge of the $p^{\gamma}$. It is the latter that we can explicitly give. Practically all the results announced here follow from the knowledge of the $p^{r}$. However, one shows that the determination of the $p^{\gamma}$ reduces to the split rank 1 case.

5. The split rank one case and 2-transitivity on a unit sphere. 1. In this section we shall assume that $\operatorname{dim} \mathfrak{a}=1$. We first need an explicit description of $\Gamma$. We find that the elements of $\Gamma$ are parameterized by a pair of integers $i(\gamma)$ and $j(\gamma)$ in a manner now to be described.

Let $8_{\mathrm{C}} \subseteq \mathrm{g}_{\mathrm{C}}$ be a normal principal TDS in the sense of [7] which is stable under conjugation over $\mathfrak{g}$, and let $z=\mathfrak{g} \cap \varepsilon_{\mathrm{C}}$. Let $S_{\theta}$ be the intersection of $G_{\theta}$ with the subgroup of the adjoint group of $g_{C}$ corresponding to ${ }^{8} \mathrm{C}$. The group $S_{\theta}$ is centerless and is never connected. In fact if $t$ is the one dimensional algebra given by $t=g \cap$ and $T_{\theta}$ is the subgroup of $S_{\theta}$ normalizing $t$ then $T_{\theta}$ is isomorphic to the orthogonal group $O(2, R)$.

Now let $b \in T_{\theta}$ be a fixed element not in the identity component of 
$T_{0}$. Then $b^{2}=1$. The subspace $[t, p]$ is stable under $b$ and we let $\mathfrak{b}$ be the +1 eigenspace of $b$ in $[t, p]$.

REMARK 4. We shall not use the fact but it can be shown that the only possible dimensions of $b$ are $1,2,4$ or 8 . Moreover there is a natural structure of a division algebra on $\delta$ with the identity lying in the one dimensional space $\mathfrak{b} \cap \bar{z}$. Of course the division algebras in the four cases are

(1) the real numbers,

(2) the complex numbers,

(3) the quaterions and

(4) the Cayley numbers.

They occur, respectively, in the case when $G$ is a suitable real form of

(1) the complex orthogonal group,

(2) the complex special unitary group,

(3) the complex symplectic group and

(4) $F_{4}$.

One also can show that $\operatorname{dim} \delta=\operatorname{dim} \mathfrak{g}^{2 \alpha}+1$ where $(\alpha)=\Lambda_{+}^{1}$ which is of course consistent with the known fact that the $\operatorname{dim} \mathrm{g}^{2 \phi}$ for any root $\phi$ takes on only the values $0,1,3,7$.

5.2. Now let $\mathrm{SO}(\mathfrak{b})$ and $\mathrm{O}(\mathfrak{d})$ be, respectively, the special orthogonal group and orthogonal groups operating on $b$ relative to the restriction of the Killing form to $b$. Consider the (complex) symmetric algebra $S(\delta)=S$ over $\delta$ and its homogeneous components $S_{k}$.

Of course the action of $O(\mathfrak{b})$ on $\mathfrak{b}$ extends to a group of automorphisms of $\delta$. The homogeneous irreducible components of $O(\delta)$ on $S$ are of course classical and well known. They are described as follows: Let $q$ be the nonzero (quadratic) invariant of $\mathrm{O}(\mathfrak{D})$ in $\delta_{2}$ and for any $j$ let $L_{j} \subseteq \delta_{j}$ be the space of harmonic elements in $\delta_{j}$ (harmonic polynomials when $\delta$ is regarded as the polynomial ring on the dual $\delta^{\prime}$ ).

Of course $L_{j}=0$ if $j \geqq 2$ when $\operatorname{dim} \mathfrak{b}=1$. In what follows $i$ and $j$ take all nonnegative integral values except when $\operatorname{dim} \delta=1$ (orthogonal case) where $j$ is restricted to 0 and 1 . With such a restriction the most general homogeneous irreducible component of $\mathrm{O}(\mathfrak{d})$ on $\mathfrak{S}$ is uniquely of the form $q^{i} L_{j}$. For $\mathrm{SO}(\delta)$ the same statement is true except that when $\operatorname{dim} \delta=2$ (unitary case) the two dimensional space $L_{j}, j>0$, is uniquely a direct sum of two irreducible components $L_{j}^{+}$ and $L_{j}^{-}$and hence the most general homogeneous irreducible component of $\mathrm{SO}(\boldsymbol{b})$ on $S$ is uniquely of the form $q^{i} L_{0}, q^{i} L_{j}^{+}$or $q^{i} L_{j}^{-}$.

Now let $J$ be the centralizer of $T_{\theta}$ in $K_{\theta}$. Clearly $\delta$ is stable under $J$ inducing a map $J \rightarrow \mathrm{O}(\mathfrak{b})$ and hence $S$ is naturally a $J$-module.

A crucial fact is given in the second paragraph of 
THEOREM 3. The group $J$ is connected and hence its action on $b$ induces a map $J \rightarrow \mathrm{SO}(\boldsymbol{b})$. Moreover, if $\operatorname{dim} \delta=1,2$ this map is surjective and if $\operatorname{dim} d>2$ then $J$ is 2-transitive on the unit sphere of $\delta$ (e.g. if $\operatorname{dim} d=8$ then $J$ operates as Spin 7).

Furthermore (as a consequence of the 2-transitivity) in all cases the homogeneous irreducible components of $J$ on $s$ are exactly the same as the homogeneous irreducible components of $\mathrm{SO}(\mathfrak{b})$ on $\mathrm{S}$. That is, the $q^{i} L_{j}\left(q^{i} L_{j}^{+}, q^{i} L_{j}^{-}\right.$and $q^{i} L_{0}$ if $\left.\operatorname{dim} \delta=2\right)$ are all irreducible and, when of the same degree, are inequivalent under the action of $J$.

5.3. Now in it there exists a unique, up to sign, element $z$ such that the eigenvalues of ad $z \mid \varepsilon_{c}$ are $0, \pm 1$. If $V$ is any finite dimensional $K_{\theta}$ module it is of course a $\mathfrak{l}_{\mathbf{C}}$ module and it follows easily that the eigenvalues of $z$ in $V$ are integers.

The structure of $\Gamma$ arises from

THEOREM 4. Let $\gamma \in \Gamma$ be arbitrary and let $k$ be the highest eigenvalue of $z$ operating in $V_{\gamma}$ and let $V_{\gamma}(z)$ be the corresponding eigenspace. Then $V_{\gamma}(z)$ is stable and irreducible under the action of $J$. Moreover $k$ is a nonnegative integer and there exists a unique J-irreducible component $Y_{\gamma} \subseteq S_{k}$ which is equivalent to the $J$-module $V_{\gamma}(z)$. Furthermore the correspondence $\gamma \rightarrow Y_{\gamma}$ thus set $u p$ between $\Gamma$ and the set of all homogeneous irreducible components of $J$ on $S$ is a bijection.

5.4. Now if $\gamma \in \Gamma$ then $Y_{\boldsymbol{\gamma}}$ by Theorem 4 is necessarily in any case a subspace of $q^{i} L_{j}$ for a unique $i$ and $j$. We put $i=i(\gamma)$ and $j=j(\gamma)$ defining the two parameters mentioned in the beginning of $\$ 5$. Note that $k=2 i(\gamma)+j(\gamma)$ where $Y_{\gamma} \subseteq s_{k}$.

Now $\Lambda_{+}^{1}$ has only one element $\alpha$ and hence there is a unique element $w \in \mathfrak{a}$ such that $\langle\alpha, w\rangle=1$. Thus $U(\mathfrak{a})$ is the polynomial ring in $w$ and hence if $p \in U(\mathfrak{a})$ we may write $p=c_{0} \Pi_{i=1}^{n}\left(w+c_{i}\right)$ where $c_{j} \in \mathbf{C}$.

We can now state

ThEOREM 5. Let $\gamma \in \Gamma$ and let $i=i(\gamma), j=j(\gamma)$. Then up to a nonzero scalar multiple

$$
\begin{aligned}
p^{\gamma}= & {[w(w+2) \cdots(w+2(i+j)-2)] } \\
\cdot & {[(w-s+1)(w-s+3) \cdots(w-s+2 i-1)] }
\end{aligned}
$$

where $s=\operatorname{dim} \mathfrak{g}^{2 \alpha}(=\operatorname{dim} \delta-1$ in the notation of $\$ 5.1$ ).

Any element $\lambda$ in $\mathfrak{a}_{\mathrm{C}}^{\prime}$ is of the form $c \alpha$ where $c \in \mathbf{C}$ and any $\lambda \in \mathfrak{a}^{\prime}$ (the real elements) is of the form $t \alpha$ where $t \in R$. The ordering in $\mathfrak{a}^{\prime}$ is of course consistent with the natural ordering $R$. An element $\lambda \in a_{c}^{\prime}$ is called a root of $p^{\gamma}$ if $p^{\gamma}(\lambda)=0$. 
REMARK 5. Note that by Theorem 5 all the roots of $p^{\gamma}$ are not only real but of the form $n \alpha$ where $n$ is an integer. Furthermore $n$ is in fact an even integer if $s \neq 0$ since $s \neq 0$ implies $s$ is odd (it is restricted to 1,3 or 7 ).

Next note that the maximal root of $p^{r}$ is 0 when $s=0$ and $s-1$ when $s>0$. Since $\rho=\left(s+\operatorname{dim} \mathfrak{g}^{\alpha} / 2\right) \alpha$ it follows that $\rho$ is larger than the largest root and in fact between the two there is an interval of "width" $\operatorname{dim} \mathrm{g}^{\alpha} / 2$ if $s=0$ and $\operatorname{dim} \mathrm{g}^{\alpha} / 2+1$ if $s>0$.

Now $g^{\gamma}(\lambda)=\overline{p^{\gamma}}(-\lambda+2 \rho)$. But since $p^{\gamma}$ is real it follows that $q^{\gamma}$ is obtained by simply reflecting $p^{\gamma}$ through $\rho$.

CoRollary to Theorem 5. Using the notation of Theorem 5 , if $p^{\gamma}$ is normalized so that the scalar multiple is 1 one has, where $m=\operatorname{dim} \mathfrak{g}^{\alpha} / 2$ $+s$,

$$
\begin{aligned}
q^{\gamma}=(-1)^{2 i+j}[(w-2 m)(w-2 m-2) & \cdots \\
\cdot[(w-2 m+s-1)(w-2 m+s-3) & \cdots \\
& \cdot(w-2 m+s-2 i+1)] .
\end{aligned}
$$

REMARK 6. Since $r^{\gamma}=q^{\gamma} p^{\gamma}$ one obtains an explicit expression for $r^{\gamma}$ and like $p^{\gamma}$ all the roots of $r^{\gamma}$ are real and of the form $n \alpha$ where $n$ is an integer and if $s>0, n$ is necessarily an even integer.

One notes also that independent of $\gamma, r^{\gamma}$ has no root of the form $\rho+c \alpha$ where $|\operatorname{Re} c|<\operatorname{dim} \mathfrak{g}^{\alpha} / 2$ if $s=0$ and $|\operatorname{Re} c|<\operatorname{dim} \mathfrak{g}^{\alpha} / 2+1$ if $s>0$. This set is what we have called the critical strip.

5.5. The situation in the case at hand $(\operatorname{dim} \mathfrak{a}=1)$ is even nicer than indicated by the last two results. Not only do we know $p^{\gamma}, q^{\gamma}$ and $r^{\gamma}$, but also, $P^{\gamma}, Q^{\gamma}$ and $R^{\gamma}$. This is a consequence of

THEOREM 6. In case $\operatorname{dim} \mathfrak{a}=1$ (our present assumption) one has $l(\gamma)=1$ for all $\gamma$. Thus $P^{\gamma}=p^{\gamma}, Q^{\gamma}=q^{\gamma}$ and $R^{\gamma}=r^{\gamma}$.

The proof of Theorem 6 comes out in the course of proving Theorem 4.

6. The determination of $p^{\gamma}, q^{\gamma}$ and $r^{\gamma}$ in the general case. 1. We now return to the general case, that is, no assumptions are made about $\operatorname{dim} \mathfrak{a}$.

Let $F$ be the group (of order $2^{r}, r=\operatorname{dim} \mathfrak{a}$ ) of all elements of order 2 in that subgroup of the adjoint group of $g_{C}$ whose Lie algebra is a . One has $F \subseteq K_{\theta}$.

Now for any $\phi \in \Lambda_{+}^{1}$ let $g_{\phi}$ be the commutator subalgebra of the reductive subalgebra $\mathfrak{a}+\mathfrak{m}+\sum_{j=-2}^{2} \mathfrak{g}^{j_{\phi}}$. Let $G_{\phi}$ be the subgroup of $G_{\theta}$ generated by $F$ and the connected subgroup (normalized by $F$ ) of 
$G_{\theta}$ whose Lie algebra is $g_{\phi}$. The Lie algebra of $G_{\phi}$ is again $\mathfrak{g}_{\phi}$ and we may apply all the preceding results to $G_{\phi}$ instead of $G$. Here the subalgebras of $\mathfrak{g}_{\phi}$ corresponding to $\mathfrak{l}, \mathfrak{a}$ and $\mathfrak{n}$ are just the intersection of these algebras with $\mathfrak{g}_{\phi}$ and are denoted by $\mathfrak{l}_{\phi}, \mathfrak{a}_{\phi}$ and $\mathfrak{n}_{\phi}$. The subgroups corresponding to $K$ and $M$ are denoted by $K_{\phi}$ and $M_{\phi}$. Furthermore this is the split rank 1 case since $\operatorname{dim} \mathfrak{a}_{\phi}=1$ and hence also the results of $\$ 5$ apply where the role of $w$ is played by the unique element $w_{\phi} \in \mathfrak{a}_{\phi} \subseteq \mathfrak{a}$ such that $\left\langle\phi, w_{\phi}\right\rangle=1$.

Now let $K_{\phi, \theta}, M_{\phi, \theta}$ and $\Gamma_{\phi}$ play the roles for $G_{\phi}$ what $K_{\theta}, M_{\theta}$ and $\Gamma$ play for $G$. Now $K_{\phi}$ operates on $\mathfrak{g}_{\mathrm{C}}$ and its restriction to $\mathfrak{g}_{\phi, \mathrm{C}}$ induces a homomorphism $K_{\phi} \rightarrow K_{\phi, \theta}$. However, since $F \subseteq K_{\phi}$ this map is a surjection. Moreover the kernel of this map is central in $K_{\phi}$ and hence is a normal subgroup of $M_{\phi}$. But since the latter maps onto $M_{\phi, \theta}$ it follows that we can regard $\Gamma_{\phi}$ as the set of all equivalence classes of (finite dimensional, continuous) irreducible $K_{\phi}$ modules $V$ such that $V^{M_{\phi}}=0$. Also by Theorem 6 one has that $\operatorname{dim} V_{\delta}^{M} \phi=1$ for all $\delta \in \Gamma_{\phi}$.

Now for any $\delta \in \Gamma_{\phi}$ the integral functions $i(\delta)$ and $j(\delta)$ are defined (as in $\$ 5$ ) and hence $p^{\delta}$ is given by Theorem 5 with $w_{\phi}$ substituted for $w$.

It is necessary to consider a slight shift in $p^{\delta}$ (since $\phi$ is not in general a simple root in $\Lambda_{+}^{1}$ ). Let $d_{\phi}$ be the integral or half-integral scalar given by $d_{\phi}=m_{\phi}-\left\langle\rho, w_{\phi}\right\rangle$ where $m_{\phi}=\operatorname{dim} \mathrm{g}^{\phi} / 2+\operatorname{dim} \mathrm{g}^{2 \phi}\left(d_{\phi}=0\right.$ if $\phi$ is simple) and let $p^{(\delta)}$ be the polynomial in $w_{\phi}$ obtained from $p^{\delta}$ by substituting $w_{\phi}+d_{\phi}$ for $w_{\phi}$. Thus if $i=i(\delta), j=j(\delta)$ one has explicitly

$$
\begin{aligned}
p^{(\delta)}= & {\left[\left(w_{\phi}+d_{\phi}\right)\left(w_{\phi}+d_{\phi}+2\right) \cdots\left(w_{\phi}+d_{\phi}+2(i+j)-2\right)\right] } \\
\cdot & {\left[\left(w_{\phi}+d_{\phi}-s_{\phi}+1\right)\left(w_{\phi}+d_{\phi}-s_{\phi}+3\right) \cdots\right.} \\
& \left.\cdot\left(w_{\phi}+d_{\phi}-s_{\phi}+2 i-1\right)\right]
\end{aligned}
$$

where $s_{\phi}=\operatorname{dim} \mathrm{g}^{2 \phi}$. Also $q^{(\delta)}$ is obtained by substituting $-w_{\phi}+2\left\langle\rho, w_{\phi}\right\rangle$ for $w_{\phi}$ in $p^{\delta}$ and one puts $r^{(\delta)}=q^{(\delta)} p^{(\delta)}$.

6.2. Now let $\gamma \in \Gamma$ be arbitrary we wish to determine $p^{\gamma}, q^{\gamma}$ and $r^{\gamma} \in U(\mathfrak{a})$ explicitly. Now let $\phi \in \Lambda_{+}^{1}$ and since $K_{\phi} \subseteq K_{\theta}$ we can consider the $K_{\phi}$-submodule $V_{\gamma}(\phi)$ of $V_{\gamma}$ generated by $V_{\gamma}^{M_{\theta}}$. Now since $M_{\phi} \subseteq M_{\theta}$ it follows from Theorem 6 that in a complete reduction of $V_{\gamma}(\phi)$ into irreducible $K_{\phi}$ components $V_{\gamma}^{i}(\phi)$ there are exactly $l(\gamma)$ components and that each $V_{\gamma}^{i}(\phi)$ belongs to a class $\delta^{i}(\phi) \in \Gamma_{\phi}$. Thus $\gamma$ defines elements $\delta^{i}(\phi) \in \Gamma_{\phi}, i=1,2, \cdots, l(\gamma)$. We now state 
THEOREM 7. For any $\gamma \in \Gamma$ one has, up to a nonzero scalar multiple,

$$
p^{\gamma}=\prod_{\phi \in \Lambda_{+}^{1}} \prod_{i=1}^{l(\gamma)} p^{\left(\delta^{i}(\phi)\right)} .
$$

Similarly $q^{\gamma}$ and $r^{\gamma}$ are respectively the products of $q^{\left(\delta^{i}(\phi)\right)}$ and $r^{\left(\delta^{i}(\phi)\right)}$ over all $\phi \in \Lambda_{+}^{1}$ and $i=1,2, \cdots, l(\gamma)$.

REMARK 7. From the expression given for $p^{(\delta)}, q^{(\delta)}$ and $r^{(\delta)}$ one notes that all the prime factors of $p^{\gamma}, q^{\gamma}$ and $r^{\gamma}$ are polynomials of the first degree.

6.3. Theorems 1 and 2 are consequences of Theorem 7. However, there are other conclusions. Let $C$ (complex Weyl chamber) be the set of all $\lambda \in \mathfrak{a}_{\mathbf{C}}^{\prime}$ such that $\operatorname{Re}\left\langle\lambda-\rho, w_{\phi}\right\rangle \geqq 0$ for all $\phi \in \Lambda_{+}^{1}$. Every $\lambda \in \mathfrak{a}_{\mathbf{C}}^{\prime}$ is $\tilde{W}$-conjugate to an element in $C$ or its negative $-C$. As a consequence of the explicit expressions of $p^{\gamma}$ and $q^{\gamma}$ one has

Corollary to Theorem 7. If $\lambda \in C$ then $p^{\gamma}(\lambda) \neq 0$ for every $\gamma \in \Gamma$ and hence, for all, $\gamma P^{\gamma}(\lambda)$ is nonsingular.

On the other hand if $\lambda \in-C$ then $q^{\gamma}(\lambda) \neq 0$ for all $\gamma \in \Gamma$ so that $Q^{\gamma}(\lambda)$ is nonsingular and hence

$$
\operatorname{rank} R^{\gamma}(\lambda)=\operatorname{rank} P^{\gamma}(\lambda) .
$$

Now whereas the irreducible $U$-module $Z^{\lambda}$ depends only on the $\tilde{W}$-orbit of $\lambda$ this is not true of the induced module $X^{\lambda}$ or of the cyclic submodule $X_{e}^{\lambda}=U \cdot 1_{\lambda}$. As a consequence of the previous corollary and Propositions 4 and 5 one has

TheOREM 8. For any $\lambda \in C$ one has $X_{e}^{\lambda}=X^{\lambda}$ so that the induced module $X^{\lambda}$ is U-cyclic. Moreover if $Y$ is any $U$-module cyclically generated by $Y^{\mathfrak{l}}$, where $\operatorname{dim} Y^{\mathfrak{l}}=1$, then $Y$ is equivalent to a quotient of $X^{\lambda}$ for some $\lambda \in C$.

For any $\lambda \in-C$ the cyclic submodule $X_{e}^{\lambda} \subseteq X^{\lambda}$ is U-irreducible and one obtains, up to equivalence, all irreducible $U$-modules $Z$, such that $Z^{\mathfrak{l}} \neq 0$, in this way.

7. Unitary representations and the complementary series. 1 . Now let $\mathfrak{a}^{*}$ be the set of all $\lambda \in \mathfrak{a}_{\mathrm{c}}^{\prime}$ such that

(1) $\lambda$ and $-\bar{\lambda}+2 \rho$ are $\tilde{W}$-conjugate (so that the $U$-invariant Hermitian bilinear form $B_{\lambda}$ exists on $Z^{\lambda}$ ) and

(2) $B_{\lambda}$ is positive definite on $Z^{\lambda}$, (therefore inducing a pre-Hilbert structure on $Z^{\lambda}$ ).

Now let $\lambda \in \mathfrak{a}^{*}$ and let $\mathfrak{H}^{\lambda}$ be the Hilbert space completion of $Z^{\lambda}$. It is easy to see that Nelson's theorem (see [8]) applies defining a 
unique unitary representation $\pi^{\lambda}$ of $G$ in $3^{\lambda}$ whose differential induces the given $U$-module structure on $Z^{\lambda}$.

Irreducibility follows easily. Also completeness follows using wellknown results of Harish-Chandra, [2], relating the action of $g$ with that of $G$. That is, one has

Proposition 6. For any $\lambda \in a^{*}, \pi^{\lambda}$ is an irreducible unitary representation of G. Moreover $\pi^{\lambda}$ is equivalent to $\pi^{\nu}$ if and only if $\lambda$ and $\nu$ are $\tilde{W}$-equivalent. Furthermore for any $\lambda \in a^{*}$ one has $\left(H^{\lambda}\right)^{K} \neq 0$ and, up to equivalence, one thus obtains all irreducible unitary representations of $G$ on a Hilbert space $H$ such that $H^{K} \neq 0$.

7.2. The obvious question is: what is the subset $a^{*} \subseteq a_{c}^{\prime}$ ? Condition (1) for an element $\lambda \in a_{c}^{\prime}$ to lie in $a^{*}$ is easy enough to deal with. If $\mu=\lambda-\rho$ this is the condition that $\mu$ and $-\bar{\mu}$ are $W$-conjugate. That is, writing $\mu=\mu_{1}+i \mu_{2}$ where $\mu_{1}, \mu_{2} \in \mathfrak{a}^{\prime}$ then condition (1) is that there should exist $\sigma \in W$ such that $\sigma \mu_{1}=-\mu_{1}$ and $\sigma \mu_{2}=\mu_{2}$.

If condition (1) is satisfied for $\lambda \in \mathfrak{a}_{\mathrm{c}}^{\prime}$ then as we noted above the matrix $R^{r}(\lambda)=P^{\gamma}(-\bar{\lambda}+2 \rho)^{*} P^{\gamma}(\lambda)$ is Hermitian for all $\gamma \in \Gamma$ (see Proposition 5). By Proposition 5 condition (2) is that the Hermitian matrix $R^{r}(\lambda)$ should be positive semidefinite for all $\gamma \in \Gamma$.

But now if $\lambda=\rho$ then $R^{r}(\rho)=P^{\gamma}(\rho)^{*} P^{r}(\rho)$ and hence $\rho \in \mathfrak{a}^{*}$. On the other hand, if $\lambda \in \mathfrak{a}_{\mathrm{C}}^{\prime}$ only satisfies condition (1) then any element on the line segment $\lambda(t)=t \lambda+(1-t) \rho$ also satisfies condition (1) and hence $R^{r}(\lambda(t))$ is a continuous curve of Hermitian matrices. But now $\rho \in C S$, the critical strip, and if $\lambda \in \overline{\mathrm{CS}}$ (the closure of CS) then $\lambda(t) \in C S$ for all $0 \leqq t<1$ and hence, for such $t, R^{\gamma}(\gamma(t))$ is a curve of nonsingular Hermitian matrices. But $R^{\gamma}(\gamma(0))$ is positive definite and since the eigenvalues cannot change sign along $\lambda(t)$ for $0 \leqq t<1$ it follows that $R^{r}(\lambda)$ is positive semidefinite. Thus for elements $\lambda \in \overline{\mathrm{CS}}$ condition (1) is necessary and sufficient for $\lambda \in \mathfrak{a}^{*}$. That is, we have proved

THEOREM 9. Let $\lambda$ be an arbitrary element in the closure of the critical strip (see \$3). Then the irreducible U-module $Z^{\lambda}$ corresponds to a (necessarily irreducible) unitary representation of $G$ if and only if there exists $\sigma$ as in the Weyl group such that $\sigma \mu_{1}=-\mu_{1}$ and $\sigma \mu_{2}=\mu_{2}$ where $\mu_{1}, \mu_{2} \in \mathfrak{a}^{\prime}$ and $\lambda-\rho=\mu_{1}+i \mu_{2}$.

REMARK 8 . To clarify the word "corresponds" one recalls that to any unitary representation of $G$ on a Hilbert space $\mathfrak{F}$ there is induced a $U$-module structure on the space $\mathcal{F}^{\infty}$ of all $C^{\infty}$ elements in $\mathcal{H}$. We will say that a $U$-module $X$ corresponds to the given unitary representation of $G$ in case $X$ is $U$-equivalent to a $U$-submodule $Y$ of $\xi^{\infty}$ 
where $Y$ is dense in $\mathfrak{H C}^{\mathrm{C}}$. However, if $Z^{\lambda}$ corresponds to the unitary $G$ module $\operatorname{TC}$ it follows easily from [2] that

(1) the representation of $G$ is irreducible,

(2) all the elements of $Y$ are well behaved and

(3) $Y$ is the set of all $K$-finite vectors in $\mathcal{H}$.

7.3. Now among all the elements of $\overline{\mathrm{CS}}$ which satisfy (condition (1) of \$7.2) the condition of Theorem 9 there are two extreme types. That is, if $\lambda \in \overline{\mathrm{CS}}$ and $\lambda-\rho=\mu=\mu_{1}+i \mu_{2}$ then

(a) if $\mu$ is pure imaginary, i.e. if $\mu_{1}=0, \lambda$ automatically satisfies condition (1) and hence $\lambda=\rho+i \mu_{2} \in a^{*}$. Here, however, comparing with $\S 2, \pi^{\lambda}$ is just an element of the principal series ( $\pi^{\lambda}$ is equivalent to $s_{\mu_{2}}$ ) and one obtains all the elements of the principal series this way.

Next

(b) if $\mu$ is real, i.e. $\mu_{2}=0$, then condition (1) of $\$ 7.2$ reduces to the condition that $\mu$ and $-\mu$ are $W$-conjugate.

We will call the family of unitary representations $\pi^{\lambda}$, where this condition holds, the complementary series since it is a natural generalization of such a designated series when $G=\operatorname{Sl}(2, R)$. In summary let CI (the critical interval) be the set of all $\lambda \in \mathfrak{a}^{\prime}$ such that $\left\langle\lambda-\rho, w_{\phi}\right\rangle \in T_{\phi}$ for all $\phi \in \Lambda_{+}^{1}$ where $T_{\phi}=\left\{t \in R|| t \mid<\operatorname{dim} g^{\phi} / 2\right.$ if $2 \phi \in \Lambda$ and $|t|<\operatorname{dim} g^{\phi} / 2+1$ if $\left.2 \phi \in \Lambda\right\}$.

Corollary to Theorem 9. Let $\lambda \in \overline{C I}$ then the irreducible $U$ module $Z^{\lambda}$ corresponds (see Remark 7) to a unitary representation of $G$ (necessarily $\pi^{\lambda}$ ) if and only if $\mu$ and $-\mu$ are $W$-conjugate where $\mu=\lambda-\rho$.

The family of unitary representations $\pi^{\lambda}$ for such $\lambda$ is called the complementary series.

REMARK 9. Note that if $-1 \in W$ then all the elements of $\overline{C I}$ satisfy the condition of the corollary above.

In general we have not as yet been able to determine all the elements of $\mathfrak{a}^{*}$ lying outside of $\overline{\mathrm{CS}}$.

For the split rank 1 case, however, $\mathfrak{a}^{*}$ can be completely determined giving therefore in that case the set of all irreducible unitary representations of $G$ with a nonzero $K$-fixed vector. The point is that if $\operatorname{dim} a=1$ then by Theorem $6, R^{\gamma}=r^{\gamma}$ and using Remark 5 it is easy to determine the set of all $\lambda \in \mathfrak{a}_{\mathrm{C}}^{\prime}$ such that $r^{\gamma}(\lambda) \geqq 0$ for all $\gamma \in \Gamma$. It turns out that except for $\lambda=0$ or $2 \rho$ the most general such $\lambda$ lies in $\overline{\mathrm{CS}}$. But $\lambda$ satisfies condition (1) of $\$ 7.3$ if and only if $\lambda-\rho$ is either real or pure imaginary. This proves

Theorem 10. Assume $\operatorname{dim} \mathfrak{a}=1$. Then except for possibly the identity representation (which may be a member of the complementary series) 
every irreducible unitary representation of $G$ with a nonzero $K$-fixed vector is either a member of the principal series or the complementary series. Furthermore (since $-1 \in W$ ) the complementary series is defined for all $\lambda \in \overline{C I}$.

REMark 10. Note that Theorem 10 points up a single irreducible unitary representation, distinguished in the following sense: The complement of $\mathrm{CI}$ in $\overline{\mathrm{CI}}$ consists of 2 points which are conjugate under $\tilde{W}$ and hence this orbit defines a single irreducible unitary representation $\pi^{\lambda}$ of $G$. In case $\operatorname{dim} \mathfrak{g}^{2 \alpha}=0,1$ (the orthogonal and unitary case), where $(\alpha)=\Lambda_{+}^{1}$, this representation is nothing more than the trivial one. However, if $\operatorname{dim} \mathfrak{g}^{2 \alpha}=3,7$ (the symplectic and $F_{4}$ cases) this representation is not trivial and it is distinguished among all nontrivial irreducible unitary representations of $G$ with a $K$-fixed vector in that it is the only one such that the subspace of $K$-finite vectors, $Z^{\lambda}$, is not $U$-equivalent to the induced module $X^{\lambda}$, i.e. $X^{\lambda}$ is not $U$-irreducible.

One notes also that in the latter case and only in this case $\left(\operatorname{dim} g^{2 \alpha}\right.$ $=3,7)$ the identity representation is not a member of the complementary series and, hence, is in fact isolated from all other unitary representations. This should perhaps be related with recent results of Kajdan. See [4].

\section{REFERENCES}

1. François Bruhat, Sur les representation induites des groupes de Lie, Bull. Soc. Math. France 84 (1956), 97-205.

2. Harish-Chandra, Representations of a semi-simple Lie group on a Banach Space. I, Trans. Amer. Math. Soc. 75 (1953), 185-243.

3. I. M. Gel'fand and M. I. Graev, Unitary representations of the real unimodular group (principal nondegenerate series), Izv. Akad. Nauk SSSR Ser. Mat. 17 (1953), 189-248; English transl., Amer. Math. Soc. Transl. (2) 2 (1956), 147-205.

4. D. A. Kajdan, Sur les relations entre l'espace dual d'un groupe et la structure de ses sous-groupes fermés, Funkcional. Anal. i Priložen. 1 (1967), 71-74.

5. Bertram Kostant, Lie group representations on a polynomial ring, Amer. J. Math. 85 (1963), 327-404.

6. Bertram Kostant and Stephen Rallis, On representation associated with symmetric spaces, Bull. Amer. Math. Soc. 75 (1969), 884-888.

7. - On orbits associated with symmetric spaces, Bull Amer. Math. Soc. 75 (1969), 879-883.

8. Edward Nelson, Analytic vectors, Ann. of Math. (2) 70 (1959), 572-615.

9. K. R. Parthasarathy, R. Ranga Rao and V.S. Varadarajan, Representations of complex semi-simple Lie groups and Lie algebras, Ann. of Math. (2) 85 (1967), 383-429.

Massachusetts Institute of Technology, Cambridge, Massachusetts 02139 